\title{
Opinion
}

\section{Educational pathways in Paediatric Virology: Pros and cons}

\author{
IOANNIS N. MAMMAS ${ }^{1-3}$, ANNE GREENOUGH ${ }^{4,5}$, \\ MARIA THEODORIDOU ${ }^{2}$ and DEMETRIOS A. SPANDIDOS ${ }^{1}$
}

\author{
${ }^{1}$ Laboratory of Clinical Virology, School of Medicine, University of Crete, 71003 Heraklion; ${ }^{2}$ First Department of Paediatrics, \\ University of Athens School of Medicine, 11527 Athens; ${ }^{3}$ Paediatric Clinic, Aliveri, 34500 Island of Euboea, Greece; \\ ${ }^{4}$ Department of Women and Children's Health, School of Life Course Sciences, Faculty of Life Sciences and Medicine, \\ King's College London, SE5 9RS London; ${ }^{5}$ Asthma UK Centre in Allergic Mechanisms of Asthma, \\ King's College London, SE1 9RT London, UK
}

Received July 22, 2019; Accepted August 26, 2019

DOI: $10.3892 / \mathrm{etm} .2019 .7955$

The '2015 Paediatric Virology proposal' (1), which was presented during the '1st workshop on Paediatric Virology' on October, 2015, resulted in a very interesting and constructive debate on the potential role of the scientific field of neonatal and paediatric viral infections as a new paediatric subspecialty candidate. To date, the proposal, which described in detail the new subspecialty areas, including both clinical and laboratory sections, has attracted the interest of several world leading experts (2-12), who have evaluated it in detail and highlighted the value of Paediatric Virology subspecialists in the future (Table I). The value of the scientific field of Paediatric Virology is also examined in the recent third supplement issue of the Experimental and Therapeutic Medicine (13-19), which is dedicated to Paediatric Virology and is edited in the context of the '5th workshop on Paediatric Virology', which will be held in Sparta, Greece on October 12, 2019.

However, specific points of scepticism have been raised (2). Amongst these, two have been considered to be as the most critical. The first one is the limited number of paediatric virologists required in each country. This can be addressed by Paediatric Virology being kept as a special interest (4) and not scheduled as a separate paediatric subspecialty (5). The Paediatric Virology subspecialty training should be offered by international centres of excellence, which would offer trainees the opportunity to be exposed to the highest case mix, while supervised by internationally renowned educationalists and researchers. A balance between subspecialisation and general knowledge should always be sustained (7).

Correspondence to: Professor Demetrios A. Spandidos, Laboratory of Clinical Virology, School of Medicine, University of Crete, 71003 Heraklion, Greece

E-mail: spandidos@spandidos.gr

Key words: Paediatric Virology, medical education, educational pathways
The second issue is the scientific background of paediatric virologists; should their medical core specialization be Microbiology, Internal Medicine or Paediatrics? Fig. 1 presents these different pathways. The supporters of the first two options are based on the current practice, where Microbiology or Internal Medicine consultants develop a special interest in Virology. We suggest that this subspecialisation should be included in Paediatrics (20). Currently, the paediatric orientated clinical training in Paediatric Virology can be undertaken by entering subspecialty training in paediatric infectious diseases (PID) or being a general paediatrician and undertaking a special interest (SPIN) module in infectious diseases or a higher degree, e.g., Master of Sciences (MSc), Doctor of Philosophy (PhD), Master of Research (MRes), or a Postgraduate Diploma in PID $(3,4,15)$. In the future, to optimize care for children requires state-of-the-art educated neonatologists and paediatricians on viral infections and paediatric virologists, who will be skilled in highly demanded diagnostic and therapeutic decisions for neonates and children with possible viral infections (20). We believe that the implementation of the '2015 Paediatric Virology proposal' into medical education and clinical practice will be a step forward in this direction.

\section{Acknowledgements}

This article is published in the third supplement issue of the Experimental and Therapeutic Medicine, which is dedicated to Paediatric Virology. This edition is performed in the context of the '5th workshop on Paediatric Virology' (Sparta, Greece, October 12, 2019) organized by the Paediatric Virology Study Group (PVSG) and supported by the Department of Clinical Virology of the University of Crete School of Medicine and the First Department of Paediatrics of the University of Athens School of Medicine. We would like to thank all the members of the PVSG for their valuable comments and corrections.

\section{References}

1. Mammas IN, Greenough A, Theodoridou M and Spandidos DA: Paediatric Virology: A new paediatric subspecialty? A proposal at the Workshop on Paediatric Virology, Athens, October 10 2015. Exp Ther Med 11: 3-5, 2016. 
Table I. Pros and cons of the '2015 Paediatric Virology proposal', which presented Paediatric Virology as a new separate paediatric subspecialty candidate.

Pros

New and old viruses as causative agents of emerging epidemics

New vaccines under investigation

New anti-viral agents in the paediatric clinical practice

Complexity of the special group of patients

New technology methods in neonatal and paediatric care

Increased clinical, research and educational needs

\section{Cons}

Subspecialty or special interest?

Jobs limitations

Clinical or basic sciences research orientation?

Subspecialty of Paediatrics or Microbiology or Internal Medicine?

Subspecialisation educational problems of the past

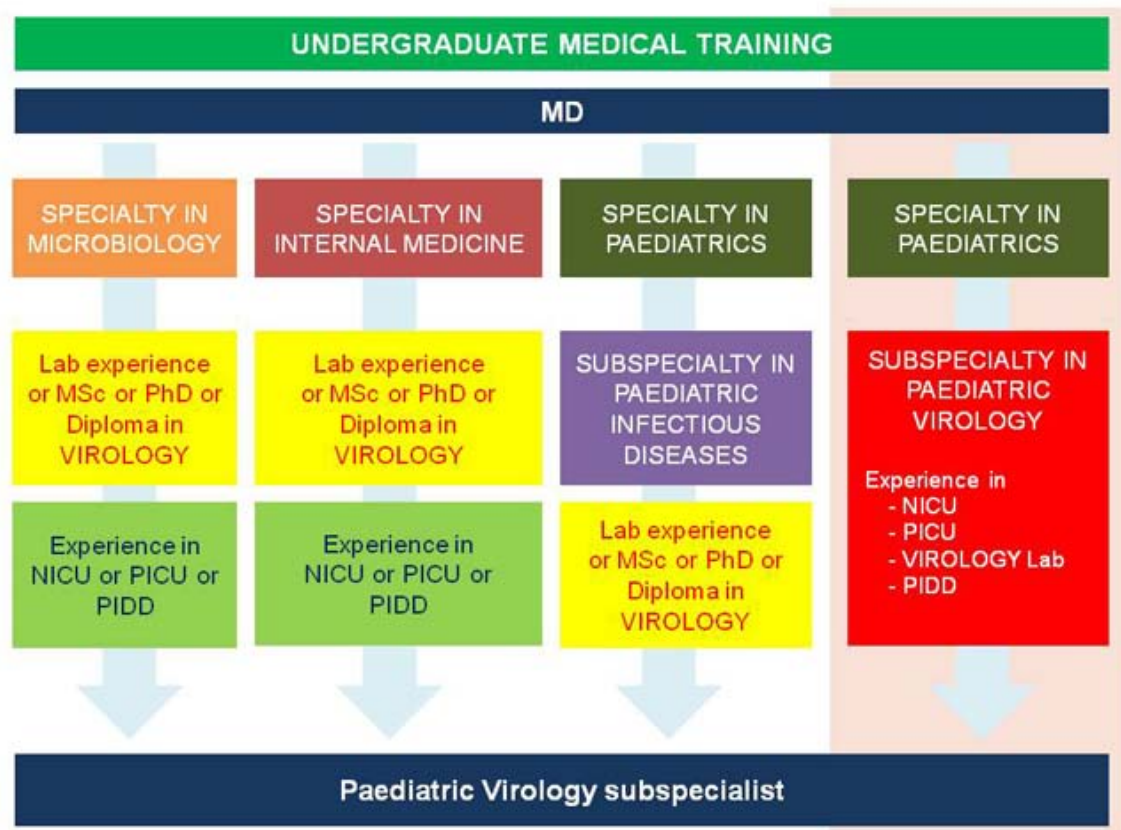

Figure 1. Different educational pathways in Paediatric Virology. MD, medical degree; Lab, laboratory; PhD, Diploma of Philosophy; MSc, Master of Sciences; NICU, Neonatal Intensive Care Unit; PICU, Paediatric Intensive Care Unit; PIDD, Paediatric Infectious Diseases Department.

2. Mammas IN and Spandidos DA: The subspecialty of Paediatric Virology: A 'mosaic tile' in future Paediatrics. Exp Ther Med 12: 539-540, 2016.

3. Mammas IN and Spandidos DA: The educational challenge of Paediatric Virology: An interview with Professor of Neonatology Anne Greenough. Exp Ther Med 14: 3332-3334, 2017.

4. Mammas IN and Spandidos DA: The future of medical education in neonatology, paediatrics and paediatric virology: An interview with Professor Alan Michael Weindling, Professor of Perinatal Medicine at the University of Liverpool. Exp Ther Med 16: 2805-2808, 2018.

5. Mammas IN and Spandidos DA: Paediatric Virology as a new educational initiative: An interview with Nobelist Professor of Virology Harald zur Hausen. Exp Ther Med 14: 3329-3331, 2017.

6. Mammas IN and Spandidos DA: The proposal of Paediatric Virology and its perspectives: An interview with Professor of Paediatrics Maria Theodoridou. Exp Ther Med 14: 3338-3340, 2017.
7. Mammas IN and Spandidos DA: The innovation of the subspecialty of Paediatric Virology: An interview with Research Professor of Molecular Virology Anna Kramvis. Exp Ther Med 14: 3335-3337, 2017.

8. Mammas IN and Spandidos DA: Paediatric virology as a candidate of paediatric subspecialisation: An interview with Assistant Professor of Molecular Microbiology-Virology Angeliki Melidou. Exp Ther Med 16: 2795-2798, 2018.

9. Mammas IN and Spandidos DA: The philosophy of paediatric teaching: An interview with Dr Nikolaos Myriokefalitakis, former Clinical Director of the 'Penteli' Children's Hospital in Athens (Greece). Exp Ther Med 16: 2799-2802, 2018.

10. Mammas IN and Spandidos DA: Neonatology, paediatrics and paediatric virology on a British island: An interview with neonatologist Dr Prakash Thiagarajan (Isle of Man). Exp Ther Med 16: 2790-2794, 2018. 
11. Mammas IN and Spandidos DA: Evaluating the proposal of paediatric virology: An interview with Professor Tina Dalianis, Professor of Tumour Virology at Karolinska Institutet. Exp Ther Med 16: 2785-2789, 2018.

12. Mammas IN and Spandidos DA: Spinal muscular atrophy type I and the dual role of viruses: An interview with Professor Basil T. Darras, Professor of Neurology (Pediatrics) at Harvard Medical School. Exp Ther Med 15: 3673-3679, 2018.

13. Mammas IN, Theodoridou M and Spandidos DA: The wisdom and eudaimonia of Paediatrics: An interview with Professor George P. Chrousos, Professor of Paediatrics at the University of Athens, Greece. Exp Ther Med 18: 3217-3220, 2019.

14. Mammas IN: Clinical Virology research and medical education in Greece: An interview with Professor Demetrios A. Spandidos, Professor of Clinical Virology at the University of Crete in Greece. Exp Ther Med 18: 3221-3225, 2019.

15. Mammas IN and Spandidos DA: Paediatric Virology and respiratory syncytial virus: An interview with Honorary Senior Lecturer in Paediatric Infectious Diseases Dr Simon B. Drysdale (St. George's, University of London, UK). Exp Ther Med 18: 3226-3230, 2019.

16. Mammas IN and Spandidos DA: Advancing challenges in Paediatric Virology: An interview with Professor Barbara A. Rath, Co-founder and Chair of the Vienna Vaccine Safety Initiative. Exp Ther Med 18: 3231-3237, 2019.
17. Mammas IN and Spandidos DA: The perspectives and the challenges of Paediatric Radiology: An interview with Dr Georgia Papaioannou, Head of the Paediatric Radiology Department at the 'Mitera' Children's Hospital in Athens, Greece. Exp Ther Med 18: 3238-3242, 2019.

18. Spandidos DA: Paediatric Virology and innovation in medical education: An interview with Dr Ioannis N. Mammas, Consultant Paediatrician on the island of Euboea (Greece) and Coordinator of the Paediatric Virology Study Group. Exp Ther Med 18: 3243-3247, 2019.

19. Mammas IN, Koutsaftiki C, Papatheodoropoulou A and Spandidos DA: Mache Papanicolaou (1890-1982), the dedicated companion of the great benefactor: An interview with Dr Julie Kokkori, one of the only living relatives of Dr George N. Papanicolaou. Exp Ther Med 18: 3248-3251, 2019.

20. Mammas IN, Greenough A, Theodoridou M and Spandidos DA: Does Europe need paediatric virologists? Exp Ther Med 16: 2783-2784, 2018

This work is licensed under a Creative Commons Attribution-NonCommercial-NoDerivatives 4.0 International (CC BY-NC-ND 4.0) License. 